\title{
SIMILARITY SOLUTIONS OF FRACTIONAL ORDER HEAT EQUATIONS WITH VARIABLE COEFFICIENTS
}

\author{
A. ELSAID, M. S. ABDEL LATIF, AND M. MANEEA
}

Received 29 March, 2015

\begin{abstract}
In this work, we use similarity method to solve fractional order heat equations with variable coefficients. The fractional derivative is defined in Caputo sense. Two examples are presented to illustrate how problems are reduced from two-variable partial fractional differential equations to ordinary ones. Series solutions are obtained for the ordinary problems in the form of power series. Based on the obtained results, a definition for an error function with generalized coefficients is proposed in a series form and its convergence is discussed.
\end{abstract}

2010 Mathematics Subject Classification: 34A25; 35K05

Keywords: similarity method, error function with generalized coefficients, Caputo fractional derivatives

\section{INTRODUCTION}

The field of fractional calculus has attracted the interest of researchers in many fields of applied sciences such as mathematics, physics, chemistry, engineering and even finance and social sciences. This is due to the fact that several definitions have been proposed for fractional derivatives and fractional integrals and have been utilized to present more accurate models for many real life phenomena. These definitions include Riemann-Liouville definition [20], Caputo definition [2], Riesz definition [21], Riesz-Feller definition [4], and the modified Riemann-Liouville definition [14]. These definitions have been used for generalizing many existing models into the form of fractional partial differential equations (FPDEs).

Two main methods have been basically used for obtaining analytic solutions to FPDEs: the first method is using Laplace transform with Fourier transform and the second method is the separation of variables technique [20]. But recently several semi-analytic methods have been also used to present series solution to FPDEs such as Adomian decomposition method [5] and [8], homotopy analysis method [10] and [13], homotopy perturbation method [7] and [17], variational iteration method [23] and [9], and fractional differential transformation method [18] and [22].

Symmetry methods are applicable to different types of linear and non linear partial differential equations. Yet, the research on using these methods for obtaining 
solutions of FPDEs is still in the initial stage. The work reported on the RiemannLiouville fractional derivative includes [1] where scaling transformations are derived for the time-fractional heat equation to reduce it to a fractional differential equation (FDE) but with Erdelyi-Kober fractional differential operator. Also, similarity solutions for the time- fractional nonlinear conduction equations are presented in [6] to reduce the considered problems to ordinary FDEs that are solved by analytic and numerical techniques. Whereas for the fractional derivative defined in Caputo sense, symmetry properties of fractional diffusion equations are studied in [11]. Finally, the fractional derivative given by Jumarie definition is studied in [3] where the Lie group method is applied to a space-time fractional diffusion equation.

In this work, we solve variable coefficients fractional-order diffusion equations with fractional time derivative defined in Caputo sense by using similarity methods to reduce FPDEs to FDEs with the same fractional derivative. Two examples are presented where the resulting FDEs are solved via series solutions. From these solutions, a new definition for error function with generalized coefficients is proposed and its convergence is proved.

This article is arranged as follows. The definition and properties of Caputo fractional derivative are listed in section two. In section three, the technique of applying the similarity method to transform FPDEs into FDEs is illustrated. In section four, power-series solutions are obtained to the FDEs and a new definition for an error function with generalized coefficients is proposed. The conclusion of this work is summarized in section five.

\section{Fractional CAlculus}

The majority of research in fractional calculus is dedicated to the two definitions of Riemann-Liouville and Caputo. Yet, the definition proposed by Caputo has been utilized more in modeling applied problems as it has two advantages. The first advantage is that the derivative of a constant equals zero and the second one is that the initial conditions can be expressed in the form of derivatives of integer order. But with the definition of Caputo, a fractional derivative is defined for differentiable functions only.

Definition 1. A real function $f(t), t>0$, is said to be in the space $C_{\mu}, \mu \in \mathbb{R}$, if there exists a real number $p>\mu$, such that $f(t)=t^{p} f_{1}(t)$, where $f_{1}(t) \in C(0, \infty)$, and it is said to be in the space $C_{\mu}^{m}$ if $f^{m} \in C_{\mu}, m \in \mathbb{N}$.

Definition 2. The Riemann-Liouville fractional integral operator of order $\alpha \geq 0$ of a function $f(t) \in C_{\mu}, \mu \geq-1$ is defined as

$$
\left\{\begin{array}{l}
J^{\alpha} f(t)=\frac{1}{\Gamma(\alpha)} \int_{0}^{t}(t-\tau)^{\alpha-1} f(\tau) d \tau, \alpha>0, t>0, \\
J^{0} f(t)=f(t) .
\end{array}\right.
$$


The operator $J^{\alpha}$ satisfies the following properties. For $f \in C_{\mu}, \mu \geq-1, \alpha, \beta \geq 0$ and $\gamma>-1$ :

1. $J^{\alpha} J^{\beta} f(t)=J^{\alpha+\beta} f(t)$,

2. $J^{\alpha} J^{\beta} f(t)=J^{\beta} J^{\alpha} f(t)$,

3. $J^{\alpha} t^{\gamma}=\frac{\Gamma(\gamma+1)}{\Gamma(\gamma+\alpha+1)} t^{\alpha+\gamma}$.

Definition 3. The fractional derivative in Caputo sense of $f(t) \in C_{-1}^{m}, m \in \mathbb{N}$, $t>0$ is defined as [20]

$$
D_{t}^{\beta} f(t)=\left\{\begin{array}{l}
J^{m-\beta} \frac{d^{m}}{d t^{m}} f(t), \\
\frac{d^{m}}{d t^{m}} f(t), \quad \beta=m .
\end{array}\right.
$$

Some basic properties of Caputo fractional derivative are:

1. If $m-1<\beta \leq m, m \in \mathbb{N}$ and $f \in C_{\mu}^{m}, \mu \geq-1$, then:

$$
D_{t}^{\beta}\left[J^{\beta} f(t)\right]=f(t),
$$

2.

$$
J^{\beta}\left[D_{t}^{\beta} f(t)\right]=f(t)-\sum_{k=0}^{m-1} f^{(k)}(0) \frac{t^{k}}{k !}, t>0 .
$$

3.

$$
D_{t}^{\beta} t^{\gamma}=\frac{\Gamma(\gamma+1)}{\Gamma(\gamma-\beta+1)} t^{\gamma-\beta}
$$

For more details on Caputo fractional derivative definition and its properties see $[16,19,20]$.

\section{SimilARITY METHOD SOLUTION}

In this section, we illustrate the technique for using similarity methods in solving fractional-order diffusion equations with Caputo definition for fractional time derivatives. The technique is illustrated by two examples. The corresponding integer-order problems of these examples can be used in modeling heat conduction with variable heat capacity and thermal conductivity of the heat conductor (see for example [12]).

\section{Problem 1}

Consider the fractional order heat equation with variable coefficients

$$
k t^{\alpha+2} \frac{\partial^{\alpha} u}{\partial t^{\alpha}}=\frac{\partial^{2} u}{\partial x^{2}}, 0<\alpha \leq 1,
$$

with the initial condition $u(x, 0)=0$. Here, $\frac{\partial^{\alpha} u}{\partial t^{\alpha}}$ denotes the partial fractional derivative of order $\alpha$ of $u=u(x, t)$ with respect to the time variable $t$ in the Caputo sense and $k$ is an arbitrary constant. To solve equation (3.1), first we perform its scaling transformation using similarity methods, see [6] and [15]. Consider the new 
independent and dependent variables denoted by $\bar{t}, \bar{x}$, and $\bar{u}$ defined in the following way

$$
t=\lambda \bar{t}, \quad x=\lambda^{p} \bar{x}, \quad u=\lambda^{q} \bar{u},
$$

where $\lambda$ is called the scaling parameter and $p$ and $q$ are arbitrary constants, to be determined such that equation (3.1) remains invariant under this transformation. Using Caputo definition (2.2), one may easily verify that

$$
\begin{aligned}
\frac{\partial^{\alpha} u}{\partial t^{\alpha}} & =\frac{1}{\Gamma(1-\alpha)} \int_{0}^{t} \frac{1}{(t-\tau)^{\alpha}} \frac{\partial u(x, \tau)}{\partial \tau} d \tau \\
& =\frac{1}{\Gamma(1-\alpha)} \int_{0}^{\bar{t}} \frac{\lambda^{q}}{(\lambda \bar{t}-\lambda \bar{\tau})^{\alpha}} \frac{\partial \bar{u}(\bar{x}, \bar{\tau})}{\partial \bar{\tau}} d \bar{\tau} \\
& =\lambda^{q-\alpha} \frac{\partial^{\alpha} \bar{u}}{\partial \bar{t}} .
\end{aligned}
$$

where $\tau=\lambda \bar{\tau}$, also

$$
\frac{\partial^{2} u}{\partial x^{2}}=\lambda^{q-2 p} \frac{\partial^{2} \bar{u}}{\partial \bar{x}^{2}} .
$$

Hence by substituting equations (3.3) and (3.4) into equation (3.1), we get

$$
k \lambda^{q+2} \frac{\partial^{\alpha} \bar{u}}{\partial \bar{t}^{-\alpha}}=\lambda^{q-2 p} \frac{\partial^{2} \bar{u}}{\partial \bar{x}^{2}} .
$$

From equation (3.5), it is clear that by setting $p=-1$ then equation (3.1) is invariant under transformation (3.2). The characteristic equation associated with transformation (3.2) is given by

$$
\frac{d u}{q u}=\frac{d x}{-x}=\frac{d t}{t} .
$$

This shows that $u(x, t)$ can be expressed as

$$
u(x, t)=x^{-q} f(\zeta),
$$

where $\zeta=x t$.

By using formula (3.7) and again using Caputo definition (2.2) for $0<\alpha \leq 1$, we have

$$
\begin{aligned}
\frac{\partial^{\alpha} u}{\partial t^{\alpha}} & =\frac{1}{\Gamma(1-\alpha)} \int_{0}^{t} \frac{1}{(t-\tau)^{\alpha}} \frac{\partial u(x, \tau)}{\partial \tau} d \tau \\
& =\frac{1}{\Gamma(1-\alpha)} \int_{0}^{\zeta} \frac{x^{-q} f^{\prime}(\zeta) x}{\left(\frac{\zeta}{x}-\frac{y}{x}\right)^{\alpha}} x^{-1} d y \\
& =\frac{1}{\Gamma(1-\alpha)} \int_{0}^{\zeta} \frac{x^{-q} f^{\prime}(\zeta)}{x^{-\alpha}(\zeta-y)^{\alpha}} d y \\
& =x^{-q+\alpha} \frac{d^{\alpha} f}{d \zeta^{\alpha}} .
\end{aligned}
$$


where $y=x \tau$. And

$$
\frac{\partial^{2} u}{\partial x^{2}}=x^{-q-2} \zeta^{2} \frac{d^{2} f}{d \zeta^{2}}-2 q \zeta \frac{d f}{d \zeta}+(q)(q+1) f(\zeta) .
$$

Putting $q=0$ and substituting equations (3.8) and (3.9) into equation (3.1), the resulting ordinary FDE is given by

$$
k \zeta^{\alpha} \frac{d^{\alpha} f}{d \zeta^{\alpha}}=\frac{d^{2} f}{d \zeta^{2}}
$$

with the initial condition $f(0)=0$.

\section{Problem 2}

Consider the fractional order heat equation with variable coefficients

$$
k t^{\alpha+2} \frac{\partial^{\alpha} u}{\partial t^{\alpha}}=2 x^{3} \frac{\partial u}{\partial x}+x^{4} \frac{\partial^{2} u}{\partial x^{2}}, 0<\alpha \leq 1,
$$

with the initial condition $u(x, 0)=0$. On the same manner, we use the similarity transformation variables

$$
t=\lambda^{n} \bar{t}, \quad x=\lambda^{p} \bar{x}, \quad u=\lambda^{q} \bar{u} .
$$

where $p, q$, and $n$ are arbitrary constants. Then we have

$$
\begin{aligned}
\frac{\partial^{\alpha} u}{\partial t^{\alpha}} & =\frac{1}{\Gamma(1-\alpha)} \int_{0}^{t} \frac{1}{(t-\tau)^{\alpha}} \frac{\partial u(x, \tau)}{\partial \tau} d \tau \\
& =\frac{1}{\Gamma(1-\alpha)} \int_{0}^{\bar{t}} \frac{\lambda^{q}}{\left(\lambda^{n} \bar{t}-\lambda^{n} \bar{\tau}\right)^{\alpha}} \frac{\partial \bar{u}(\bar{x}, \bar{\tau})}{\partial \bar{\tau}} d \bar{\tau} \\
& =\lambda^{q-n \alpha} \frac{\partial^{\alpha} \bar{u}}{\partial \bar{t}^{-\alpha}} .
\end{aligned}
$$

where $\tau=\lambda^{n} \bar{\tau}$, also

$$
\begin{aligned}
\frac{\partial u}{\partial x} & =\lambda^{q-p} \frac{\partial \bar{u}}{\partial \bar{x}}, \quad \text { and } \\
\frac{\partial^{2} u}{\partial x^{2}} & =\lambda^{q-2 p} \frac{\partial^{2} \bar{u}}{\partial \bar{x}^{2}} .
\end{aligned}
$$

By substituting equations (3.13) and (3.14) into equation (3.11), we get

$$
k \lambda^{2 n-4 p+q} \bar{t}^{\alpha+2} \frac{\partial^{\alpha} \bar{u}}{\partial \bar{t}^{\alpha}}=2 \bar{x}^{3} \lambda^{q-2 p} \frac{\partial \bar{u}}{\partial \bar{x}}+\bar{x}^{4} \lambda^{q-2 p} \frac{\partial^{2} \bar{u}}{\partial \bar{x}^{2}} .
$$

From equation (3.15), it is clear that by setting $n=p$ then equation (3.11) is invariant under transformation (3.12). The characteristic equation becomes

$$
\frac{d u}{q u}=\frac{d x}{p x}=\frac{d t}{p t} .
$$


By solving the characteristic equation (3.16), and putting $q=0, u(x, t)$ can be expressed as

$$
u(x, t)=f(\zeta), \quad \zeta=t x^{-1} .
$$

Again, using formula (3.17) and Caputo definition (2.2) for $0<\alpha \leq 1$, we have

$$
\begin{aligned}
\frac{\partial^{\alpha} u}{\partial t^{\alpha}} & =\frac{1}{\Gamma(1-\alpha)} \int_{0}^{t} \frac{1}{(t-\tau)^{\alpha}} \frac{\partial u(x, \tau)}{\partial \tau} d \tau \\
& =\frac{1}{\Gamma(1-\alpha)} \int_{0}^{\zeta} \frac{f^{\prime}(\zeta) x^{-1}}{(x \zeta-x y)^{\alpha}} x d y \\
& =\frac{1}{\Gamma(1-\alpha)} \int_{0}^{\zeta} \frac{f^{\prime}(\zeta)}{x^{\alpha}(\zeta-y)^{\alpha}} d y \\
& =x^{-\alpha} \frac{d^{\alpha} f}{d \zeta^{\alpha}} .
\end{aligned}
$$

where $y=x^{-1} \tau$. And

$$
\begin{aligned}
\frac{\partial u}{\partial x} & =-t x^{-2} \frac{d f}{d \zeta}, \\
\frac{\partial^{2} u}{\partial x^{2}} & =t^{2} x^{-4} \frac{d^{2} f}{d \zeta^{2}}+2 t x^{-3} \frac{d f}{d \zeta} .
\end{aligned}
$$

Substituting into equation (3.11) from equations (3.18) and (3.19), the FPDE (3.11) is reduced to

$$
k \zeta^{\alpha} \frac{d^{\alpha} f}{d \zeta^{\alpha}}=\frac{d^{2} f}{d \zeta^{2}},
$$

with the initial condition $f(0)=0$ which is the ordinary FDE (3.10) obtained in first problem.

\section{POWER-SERIES SOLUTION}

To find the solution of equation (3.10) we consider the FDE of the form

$$
k \zeta^{\alpha} \frac{d^{\alpha} f}{d \zeta^{\alpha}}=\frac{d^{2} f}{d \zeta^{2}} .
$$

The solution is assumed in the power series form

$$
f(\zeta)=\sum_{n=0}^{\infty} a_{n} \zeta^{n} .
$$

By substituting series solution (4.2) into equation (4.1) and equating the coefficients of similar powers in both sides, we get

$$
a_{2 n}=0, n=0,1,2, \ldots
$$




$$
\begin{aligned}
& a_{3}=k \frac{\Gamma(2)}{3 ! \Gamma(2-\alpha)} a_{1}, \\
& a_{5}=k^{2} \frac{\Gamma(4) \Gamma(2)}{5 ! \Gamma(4-\alpha) \Gamma(2-\alpha)} a_{1},
\end{aligned}
$$

The resulting solution becomes

$$
f(\zeta)=a_{1}\left[\zeta+k \frac{\Gamma(2)}{3 ! \Gamma(2-\alpha)} \zeta^{3}+k^{2} \frac{\Gamma(4) \Gamma(2)}{5 ! \Gamma(4-\alpha) \Gamma(2-\alpha)} \zeta^{5}+\ldots\right],
$$

which has the fractional order derivative

$$
\begin{aligned}
\frac{d^{\alpha} f}{d \zeta^{\alpha}}= & a_{1}\left[\frac{1}{\Gamma(2-\alpha)} \zeta^{1-\alpha}+k \frac{\Gamma(2) \Gamma(4)}{3 ! \Gamma(2-\alpha) \Gamma(4-\alpha)} \zeta^{3-\alpha}\right. \\
& \left.+k^{2} \frac{\Gamma(2) \Gamma(4) \Gamma(6)}{5 ! \Gamma(2-\alpha) \Gamma(4-\alpha) \Gamma(6-\alpha)} \zeta^{5-\alpha}+\ldots\right] .
\end{aligned}
$$

Based on these results, we propose a definition for error function with generalized coefficients and discuss its convergence.

Definition 4. An error function with generalized coefficients is defined in the form

$$
\operatorname{erf}(\zeta ; k ; \alpha)=\frac{1}{\sqrt{\pi}} \sum_{n=0}^{\infty} a_{2 n+1} \zeta^{2 n+1},
$$

where

$$
\begin{aligned}
a_{1} & =1, \\
a_{2 n+1} & =\frac{k^{n}}{(2 n+1) !} \prod_{i=1}^{n} \frac{\Gamma(2 i)}{\Gamma(2 i-\alpha)}, n=1,2, \ldots
\end{aligned}
$$

By this definition, the solution to FDE (4.1) is given by

$$
f(\zeta)=c_{1}+c_{2} \operatorname{erf}(\zeta ; k ; \alpha),
$$

where $c_{1}$ and $c_{2}$ are arbitrary constants. Hence, in the solution of FDE (3.10) with the initial condition $f(0)=0, c_{1}$ equals zero.

Evidently, when substitute $\alpha=1$ and $k=-\lambda^{2}$ in definition (4.5)-(4.6), the obtained series is the Taylor series of the classical integer-order error function $\operatorname{erf}\left(\frac{\lambda \zeta}{\sqrt{2}}\right)$. This coincides with the fact that in this case equation (4.1) becomes the ordinary differential equation $-\lambda^{2} \zeta \frac{d f}{d \zeta}=\frac{d^{2} f}{d \zeta^{2}}$ which has the general solution of the form $f(\zeta)=c_{1}+c_{2} \operatorname{erf}\left(\frac{\lambda \zeta}{\sqrt{2}}\right)$, with $c_{1}=0$ to satisfy the initial condition $f(0)=0$. Also, when $\alpha$ approaches zero and $k=-\lambda^{2}$, in this case the obtained series is the Taylor series of the classical trigonometric sine function $\sin (\lambda \zeta)$. This coincides with the fact 
that in this case equation (4.1) becomes the ordinary differential equation $-\lambda^{2} f=$ $\frac{d^{2} f}{d \zeta^{2}}$ which has the general solution of the form $f(\zeta)=c_{1} \sin (\lambda \zeta)+c_{2} \cos (\lambda \zeta)$, with $c_{2}=0$ to satisfy the initial condition $f(0)=0$.

To check the radius of convergence of the error function with generalized coefficients defined by (4.5)-(4.6), we evaluate the limit

$$
\begin{aligned}
\lim _{n \rightarrow \infty}\left|\frac{a_{2 n+3}}{a_{2 n+1}}\right| & =\lim _{n \rightarrow \infty}\left|\frac{k \Gamma(2 n+2)}{(2 n+3)(2 n+2) \Gamma(2 n+2-\alpha)}\right| \\
& =|k| \lim _{n \rightarrow \infty}\left|\frac{\Gamma(2 n+2)}{(2 n+3)(n+1) \Gamma(2 n+2-\alpha)}\right| \\
& =0 .
\end{aligned}
$$

Hence, the series converges for all $\zeta$.

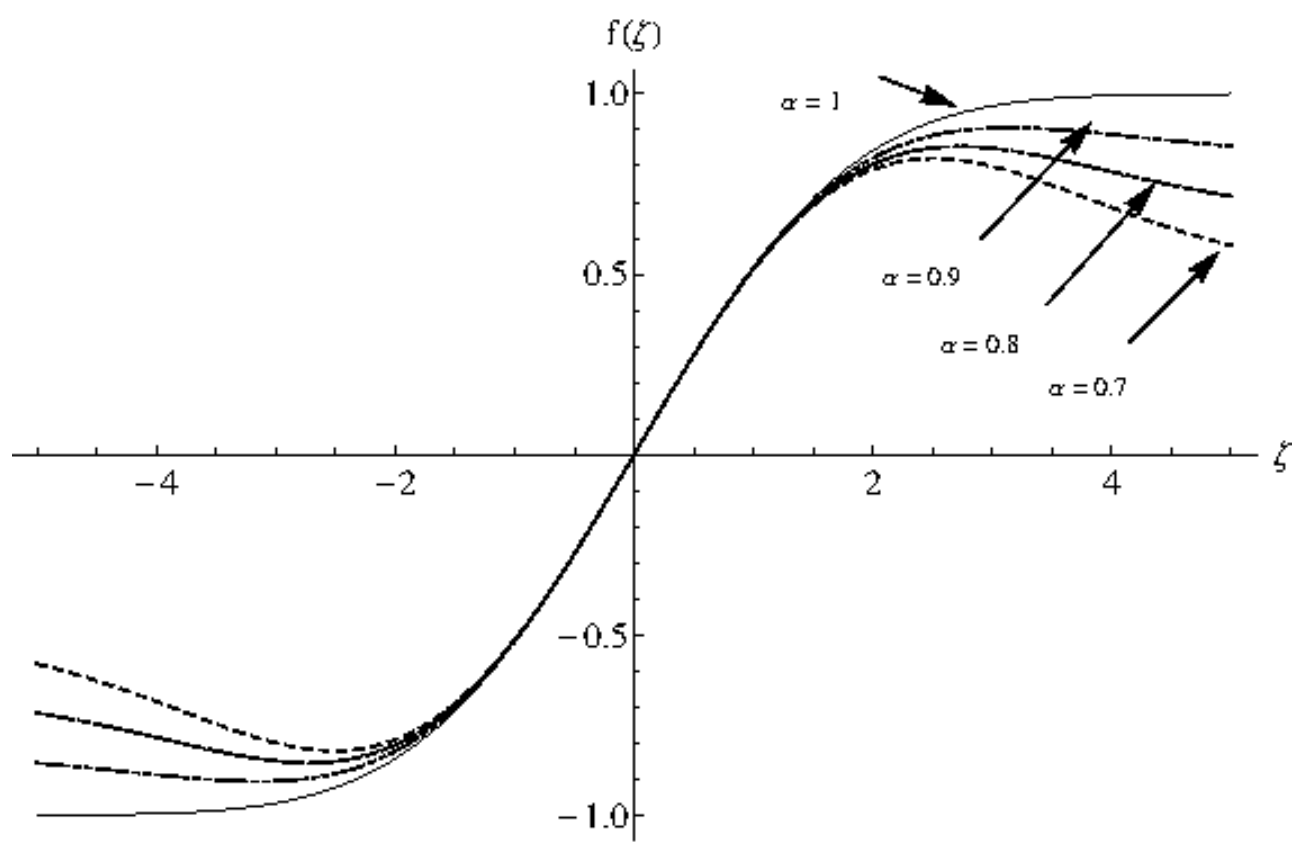

FIGURE 1 . The function $\operatorname{erf}\left(\zeta ;-\frac{1}{2} ; \alpha\right)$ at different values of $\alpha$.

Figure 1 shows the effect of changing the order of fractional derivative $\alpha$ on the behavior of the series representation of the solution function $\operatorname{erf}\left(\zeta ;-\frac{1}{2} ; \alpha\right)$ at different values of $\alpha$ (This figure is graphed with $n=40$ ). Figure 1 also illustrates that as $\alpha$ approaches one, the graph of the solution coincides with the graph of the classical error function $\operatorname{erf}\left(\frac{\xi}{2}\right)$ which is the solution of the integer-order differential equation corresponding to FDE (3.10). 


\section{Conclusion}

The similarity method is utilized to solve variable coefficients time-fractional heat equations where the fractional derivative is given in Caputo sense. Because the similarity method decreases the number of independent variables of the equation by one variable, we use it to transform the time-fractional heat equations with two independent variables into an ordinary FDE in the same fractional derivative sense. The solution to the ordinary FDE is obtained in the form of a power series which motivated a definition for what we call the error function with generalized coefficients which has an infinite radius of convergence.

\section{REFERENCES}

[1] E. Buckwar and Y. Luchko, "Invariance of a partial differential equation of fractional order under the Lie group of scaling transformations," J. Math. Anal. Appl., vol. 21, pp. 81-97, 1998, doi: 10.1006/jmaa.1998.6078.

[2] M. Caputo, "Linear model of dissipation whose Q is almost frequency dependent II, Geophys," $J$. R. Ast. Soc., vol. 13, pp. 529-539, 1967, doi: 10.1111/j.1365-246X.1967.tb02303.x.

[3] G. cheng Wu, "A Fractional Lie Group Method For Equations," Commun. Frac. Calc., vol. 1, pp. 27-31, 2010 .

[4] M. Ciesielski and J. Leszczynski, "Numerical solutions to boundary value problem for anomalous diffusion equation with Riesz-Feller fractional operator," J. Theor. Appl. Mech., vol. 44, no. 3, pp. 393-403, 2006.

[5] D. B. Dhaigude and G. A. Birajdar, "Numerical solution of system of fractional partial differential equations by discrete Adomian decomposition method," J. of Frac, Calc. and Applic., vol. 3, no. 12 , pp. 1-11, 2012.

[6] V. D. Djordjevic and T. M. Atanackovic, "Similarity solution to nonlinear heat conduction and Burgers/Korteweg-de Vries fractional equation," J. Comput. Appl. Math., vol. 222, pp. 701-714, 2008, doi: 10.1016/j.cam.2007.12.013.

[7] A. El-Sayed, A. Elsaid, I. El-Kalla, and D. Hammad, "A homotopy perturbation technique for solving partial differential equations of fractional order in finite domains," Appl. Math. and Comp., vol. 218, pp. 8329-8340, 2012, doi: 10.1016/j.amc.2012.01.057.

[8] A. El-Sayed and M. Gaber, "The Adomian decomposition method for solving partial differential equations of fractal order in finite domains," Phys. Lett. A, vol. 359, pp. 175-182, 2006, doi: 10.1016/j.physleta.2006.06.024.

[9] A. Elsaid, "The variational iteration method for solving Riesz fractional partial differential equations," Comp. and Math. with Applic. A, vol. 60, pp. 1940-1947, 2010, doi: 10.1016/j.camwa.2010.07.027.

[10] A. Elsaid, "Homotopy analysis method for solving a class of fractional partial differential equations," Commun. Nonlinear Sci. Numer. Simulat., vol. 16, pp. 3655-3664, 2011, doi: 10.1016/j.cnsns.2010.12.040.

[11] R. Gazizov, A. Kasatkin, and S. Lukashchuk, "Symmetry properties of fractional diffusion equations," Phys. Scr., vol. T136, p. 014016, 2009, doi: 10.1088/0031-8949/2009/T136/014016.

[12] M. S. Hussein, D. Lesnic, and M. I. Ivanchov, "Simultaneous determination of time-dependent coefficients in the heat equation," Comput. Math. Appl., vol. 67, pp. 1065-1091, 2014, doi: 10.1016/j.camwa.2014.01.004. 
[13] H. Jafari, A. Golbabai, S. Seifi, and K. Sayevand, "Homotopy analysis method for solving multiterm linear and nonlinear diffusion wave equations of fractional order," Comput. .Math. with Appl., vol. 59, pp. 1337-1344, 2010, doi: 10.1016/j.camwa.2009.06.020.

[14] G. Jumarie, "Tables of some basic fractional calculus formulae derived from a modified RiemannLiouville derivative for non-differentiable functions," Appl. Math. Lett., vol. 22, pp. 378-385, 2009, doi: 10.1016/j.aml.2008.06.003.

[15] R. Kandasamy and I. Muhaimin, "Scaling transformation of the effect of temperature-dependent fluid viscosity with thermophoresis particle deposition on MHD-free convective heat and mass transfer over a porous stretghing surface," Transp. Porous Med., vol. 84, pp. 549-568, 2010, doi: 10.1007/s11242-009-9519-6.

[16] M. M. Khader, N. H. Sweilam, A. M. S. Mahdy, and N. K. A. Moniem, "Numerical simulation for the fractional SIRC model and Influenza A,” Appl. Math. Inf. Sci., vol. 8, no. 3, pp. 1029-1036, 2014.

[17] S. Momani and Z. Odibat, "Homotopy perturbation method for nonlinear partial differential equations of fractional order," Phys. Lett. A, vol. 365, pp. 345-350, 2007, doi: 10.1016/j.physleta.2007.01.046.

[18] Z. Odibat and S. Momani, "A generalized differential transform method for linear partial differential equations of fractional order," Appl. Math. Lett., vol. 21, pp. 194-199, 2008, doi: 10.1016/j.aml.2007.02.022.

[19] K. B. Oldham and J. Spanier, The Fractional Calculus. New York: Academic Press, 1974.

[20] I. Podlubny, Fractional differential equations. San Diego: Academic Press, 1999.

[21] S. G. Samko, A. A. Kilbas, and O. L. Marichev, Fractional Integrals and Derivatives: Theory and Applications. New York: Gordon and Breach, 1993.

[22] A. Secer, M. A. Akinlar, and A. Cevikel, "Efficient solutions of systems of fractional PDEs by the differential transform method," Advances in Difference Equations, p. 188, 2012, doi: 10.1186/1687-1847-2012-188.

[23] V. Turut and N. Guzel, "On solving partial differential equations of fractional order by using the variational iteration method and multivariate Pade approximations," Euro. J. of Pure and Appl. Math., vol. 6, no. 2, pp. 147-171, 2013.

\section{Authors' addresses}

A. Elsaid

Mansoura university, Mathematics \& Engineering Physics Department, Faculty of Engineering, PO 35516, Mansoura,, Egypt

E-mail address: a_elsaid@mans.edu.eg

M. S. Abdel Latif

Mansoura university, Mathematics \& Engineering Physics Department, Faculty of Engineering, PO 35516, Mansoura,, Egypt

E-mail address: m_gazia@mans.edu.eg

\section{Maneea}

Modern university for technology and information, Mathematics \& Engineering Physics Department, Faculty of Engineering, Cairo,, Egypt

E-mail address: eng.marwa1981@yahoo.com 